\title{
e-Phaïstos
}

e-Phaïstos

Revue d'histoire des techniques / Journal of the history

of technology

III-1 | 2014

Varia

\section{La pensée technique de l'Académie Royale des Sciences (1699-1750)}

A new approach of thinking the techniques: the French "Académie Royale des

Sciences" (1699-1750)

\section{Bernard Delaunay}

\section{(2) OpenEdition}

Journals

Édition électronique

URL : http://journals.openedition.org/ephaistos/652

DOI : 10.4000/ephaistos.652

ISSN : 2552-0741

Éditeur

IHMC - Institut d'histoire moderne et contemporaine (UMR 8066)

Édition imprimée

Date de publication : 1 juin 2014

Pagination : 105-110

ISSN : 2262-7340

Référence électronique

Bernard Delaunay, «La pensée technique de l'Académie Royale des Sciences (1699-1750) », e-Phaïstos [En ligne], III-1 | 2014, mis en ligne le 24 novembre 2016, consulté le 15 septembre 2020. URL : http:// journals.openedition.org/ephaistos/652 


\title{
Bernard Delaunay
}

\section{La pensée technique de l'Académie royale des sciences}

\author{
Thèse de doctorat \\ Équipe d'Histoire des Techniques / IHMC \\ CNRS-Université Paris 1 Panthéon-Sorbonne \\ Soutenue le 11 décembre 2013
}

Un volume de texte (414 p.), un volume d'annexes (348 p.)

Directeur de thèse :

Anne-Françoise GARÇON, professeur, Université Paris 1 Panthéon-Sorbonne

Jury :

Simone MAZAURIC, professeur, Université Nancy 2

Hélène VERIN, chargée de recherche, CNRS, Centre Alexandre Koyré, UMR 85560

Pascal BRIOIST, professeur, Université Rabelais, Tours

Jean-Luc CHAPPEY, maître de conférences habilité à diriger des recherches, Université Paris 1 Panthéon-Sorbonne
Monsieur le Président,

Mesdames et Messieurs, membres du jury,

Comment ne pas débuter cet exposé sans évoquer un souvenir personnel datant de 1972: jeune ingénieur ayant à conduire l'installation sur un navire militaire d'un « loch à tube de Pitot » et son utilisation par les systèmes radar, j'ai demandé que l'on explique ce qu'était ce type de loch, je ne me suis même pas soucié de savoir qui était Pitot. Arrivé dans le dépouillement des registres de séances à l'année 1732, j'ai redécouvert ce type de loch et son inventeur. Grâce à l'éloge de Pitot prononcé par Grandjean de Fouchy, à sa mort en 1771, je sais maintenant qui était Pitot, et cela avec bien d'autres résultats, au terme d'un travail de recherche qui m'a passionné.

$\mathrm{Si}$, comme l'exprime Cournot, le hasard est la rencontre de deux séries causales indépendantes, alors l'origine de la thèse que j'ai l'honneur de vous présenter aujourd'hui pourrait bien être le fruit du hasard faisant se rencontrer les hommes de la Renaissance face à "l'universelle contrariété du monde » et les ingénieurs contemporains face à «l'universelle contrariété des logiciels ». En effet, s'est opéré pour moi un rapprochement, en découvrant la «Réduction en art " et en la comparant aux efforts normatifs des méthodes de développements logiciels. Cela m'a conduit, après une discussion avec Anne-Françoise Garçon, dans cette toute proche galerie Jean-Baptiste Dumas ${ }^{1}$, à 
mon sujet de Master 1. La question initiale, telle qu'elle était envisagée alors, était de rechercher des traces, des origines, de la normalisation technique à travers le mécanisme des prix de l'Académie Royale des sciences et c'est avec une certaine insouciance que j'ai entamé ce Master 1. Insouciance car j’ignorais au fond où cela allait me mener.

La question, avec le recul, était bien vague mais ce qui ne l'était pas, c'était les sources que j’allais aborder. Les registres de séances de l'Académie royale des sciences étaient là, bien disponibles, en libre-service grâce à la photocopie des microfilms réalisés par le CNRS dans les années 1960, aux archives de l'Académie, où l'accueil était presque familial. Ce n'est que sur la fin des dépouillements que j'ai pu profiter de leur mise à disposition numérique par la BNF. J'ai alors complété les tableaux de dépouillement par les numéros de pages de la version électronique, rendant très rapide des retours et des vérifications.

Les premières approches de la bibliographie, celle de l'histoire de l'institution au XVIII' ${ }^{\mathrm{e}}$ siècle, la mise en route de ce projet de recherche, avec hésitations et erreurs, m'avaient conduit à commencer un dépouillement des registres à l'année 1714. Pourquoi 1714 ? Parce que c'est l'année du testament de Rouillé de Meslay qui institue le premier prix. Evidemment, je ne pouvais rien trouver cette année-là, puisque le testament, contesté en justice, ne fut effectif qu'en 1720. Au fond, c'était encore un hasard, que la rencontre de cette erreur, avec le second mémoire d'Antoine Parent sur les machines hydrauliques présenté précisément en 1714. Mémoire non publié, Parent n'a guère de considération académique, Fontenelle se contente de le résumer et termine par cette phrase que je considère comme le déclic de ma recherche : Ce n'est plus que du calcul.

Naturellement, en avançant dans les dépouillements, je prenais conscience de la place de la technique à l'Académie essentiellement dans les examens des inventions et dans les descriptions des arts et métiers. Mais ce qui n'était pas dit dans la bibliographie que je consultais alors, la place de la technique dans l'activité académique interne, je la voyais apparaître à travers cette phrase banale pour un ingénieur, "ce n'est plus que du calcul», conclusion évidente d'une étude technique.

C'est à ce moment qu'a pris corps l'idée d'une recherche historique sur la place de la technique dans cette académie du premier XVIII ${ }^{e}$ siècle. Quelle place pour la technique, pourquoi cet intérêt des savants pour les arts, somme toute assez peu considérés par la société du temps ? Mais, au-delà de ces questions, j'en ai progressivement découvert une autre, comment les académiciens s'occupaientils de technique, comment la pensaient-ils ? Le séminaire d'Anne-Françoise Garçon sur la pensée technique, son ouvrage sur les régimes de la pensée opératoire $^{2}$, l'ouvrage d'Hélène Vérin sur la gloire des Ingénieurs 3 , celui d'Antoine Picon sur la naissance de l'ingénieur moderne ${ }^{4}$, celui de François Russo sur l'introduction à l'histoire des techniques ${ }^{5}$, celui, enfin, de Simone Mazauric sur l'histoire des sciences à l'époque moderne ${ }^{6}$, m'ont permis en particulier de clarifier les questions que je me posais. Ce questionnement était indispensable pour dépasser la simple accumulation statistique des activités techniciennes de l'Académie

Face aux milliers de pages des registres de séance, il fallait, si j'ose utiliser ce mot un peu trivial, «plonger ». La lecture en était parfois rebutante et l'attention se disperse et se perd, obligeant à des retours décourageants mais cette lecture de tous les textes à caractère technique était indispensable. Lecture ne veut pas dire, malheureusement, compréhension fine des contenus. Si j'ai pu en analyser quelques-uns, voire en refaire les calculs, les détails m'ont aussi échappés pour pas mal d'entre eux. Mais mon propos n'était pas de les comprendre tous, mais d'en tirer des classifications et des types et pour cela une compréhension globale de l'objet de l'expertise ou du mémoire s'est avérée suffisante.

Compte tenu de ce volume de sources il a donc été nécessaire de structurer le dépouillement. Cette 
structuration s'est faite à travers une base de données Microsoft Excel, je devrais dire plusieurs, regroupées progressivement et liées entre elles, enrichies, reprises, complétées. L'outil permet en effet une saisie quasi cursive des textes puis l'introduction ultérieure de critères de choix qui permettent les tris. J'ai préféré toutes ces manipulations un peu artisanales à la constitution a priori d'une base de données Microsoft Access certainement plus élégante mais dont je ne pouvais pas initialement prévoir tous les champs de données dont l'analyse serait pertinente. Cela n'a pas été sans quelques retours parfois laborieux, j’ai ainsi dépouillé deux fois les années 1700-1710 mais, dès le début du travail de doctorat, cette base été constituée, il ne restait plus, c'est une litote, qu'à achever de la remplir et à en tirer des statistiques et des synthèses.

Les premiers résultats, les premiers dépouillements, m'ont confirmé cette place de la technique, assez ignorée de l'historiographie. L'expertise sur les moyens d'essais de la qualité des poudres livrées à la marine royale en 1702-1704 montrait la rigueur du raisonnement et le soin expérimental déployés dans la rédaction d'une «instruction technique » sur la fabrication des poudres et sur l'utilisation d'une pièce d'artillerie à tir courbe, un mortier, avec un boulet calibré et une charge de poudre calibrée, pour en vérifier la portée. Le mémoire de Parent (encore lui !) mettant en équation une roue hydraulique et trouvant le maximum de l'effet, du rendement dirions-nous, en dérivant une fonction, belle et précoce application du calcul différentiel en 1704, voilà qui m'a conforté dans la nécessité du dépouillement systématique. Apparaissait alors l'importance de ces études techniques qui n'étaient mentionnées que dans les registres et les volumes annuels des Histoires et Mémoires.

Du Master 1 au doctorat, j'ai donc accumulé les années dépouillées de 1700 à 1750 en réintégrant à la fin l'année 1699, exclue qu'elle était par coquetterie statistique, puisqu'elle ne commençait qu'en février et n'avait pas l'honneur d'un registre propre. Les bornes temporelles de ma recherche 1699-1750 étaient alors fixées. L'année 1699 va de soi par le renouvellement et, pour confirmer ce moment clé, j’ai examiné les années 1680-1698, années nettement moins riches en manifestations d'une activité technicienne. Il y a bien dans l'activité académique un avant et un après 1699. Le choix de 1750 comporte une part d'arbitraire, lié à la nécessité de respecter un calendrier, raison contingente mais réelle. Mais ce tournant du siècle est aussi celui de la création des premières écoles d'ingénieurs, les Ponts et Mézières, les débuts d'une chimie et d'une métallurgie scientifique avec Hellot traduisant Schlutter ${ }^{7}$ et plus tard Gabriel Jars et ses voyages métallurgiques analysés par AnneFrançoise Garçon. Sans que l'année 1750 marque une borne aussi claire que 1699 , on peut considérer que le milieu du siècle représente une inflexion. J'ai donc voulu mettre en lumière cette première moitié du siècle. Au total donc, de 1680 à 1750, 71 années dépouillées, probablement un peu plus de 20000 pages de registres.

Ces milliers de pages saisies partiellement, réduites à des cellules de tableaux, posaient alors plusieurs séries de questions. La première, la plus générale, celle des modalités et j'ai donc commencé à trier ces activités technicienne en " externes ", c'est à dire examens d'invention et expertises, et « internes », c'est-à-dire présentation d'un mémoire technique. Ensuite venait tout naturellement la question de la typologie de ces examens et de ces études, d'où les catégories, mécaniques, naval, militaires, transports etc. Enfin, dernière question plus tardive, qui m'a obligé à une nouvelle lecture, pouvait-on mettre en lumière parmi les académiciens, des acteurs plus tournés vers la technique.

Faire des tris, définir des catégories, des modalités, cela comporte nécessairement de prendre des partis, de faire des choix. Ils peuvent naturellement être critiqués, j'ose les assumer dans la mesure où je pense qu'ils montrent une certaine 
efficacité. Une fois ces choix faits, il fallait s'y tenir avec rigueur et méthode sous peine de voir disparaitre la pertinence des statistiques. Ces statistiques un peu arides mais indispensables établissent de façon quantitative la place et les modalités des travaux de l'Académie ainsi que les domaines techniques les plus étudiés.

Deux catégories initiales donc qui n'auraient donné que deux chapitres, «Examiner» et "Etudier» mais analysant plus attentivement les textes, la durée des actions d'examen ou d'expertise, les publications, il m'est apparu clairement qu'examiner une invention d'une machine, en vue de l'approuver, était d'une toute autre nature que de rédiger une instruction sur le jaugeage des vaisseaux. Approuver un cas particulier, parfois avec réserve, laissant au fond le risque technique et économique à l'inventeur, même si les commissaires tiennent compte de ces aspects, voilà qui est différent de la résolution d'un problème technique général, engageant la responsabilité de l'Académie vis-à-vis de son administration de tutelle. Les " grandes expertises », les poudres, le jaugeage, les teintures, sont longues, débattues en séances, leurs conclusions sont imprimées comme des mémoires techniques. Dans les tâtonnements du début je me suis rendu compte que j'en classais certaines comme examen d'invention et d'autres comme études techniques, il fallait se résoudre à créer cette catégorie et créer le chapitre « Expertiser », plus bref que les autres mais, selon moi, nécessaire.

De même dans ces études techniques internes, regrouper la lecture d'une description d'un art avec un mémoire technique allait de soi, au premier abord. Mais le sens commun est vite désabusé à la fois par la place infime laissée à ces descriptions dans les Histoires annuelles et par les commentaires répétés sur l'importance des " principes » et le moindre intérêt des descriptions. Une distinction s'est alors imposée, ce qui compte aux yeux des académiciens, c'est de répondre d'abord à la question " pourquoi ça marche » et non
« comment ça marche». Les descriptions et les observations partent de l'existant pour aller vers la description, du bas vers le haut, alors que les académiciens cherchent à partir du haut, des principes, vers le bas, le détail. Le comment est laissé aux descriptions qui se fixent sur ce qui existe et en quelque sorte pourraient être assimilées aux réductions en art qui partent de l'existant pour le rédiger le mettre en ordre. Ayant identifié une inversion de l'approche entre les descriptions et les études, je me devais d'en tenir compte. Il semblait enfin difficile de ne pas mettre en regard, dans ce chapitre consacré aux descriptions des arts, le travail de l'Académie et les descriptions des arts contenues dans l'Encyclopédie de Diderot et d'Alembert.

Ainsi les quatre modalités, les quatre chapitres ont pris leur place dans un plan qui ne pouvait selon moi qu'être thématique. Parallèlement à ces classifications qui permettaient d'avoir une vue raisonnée de l'activité technicienne de l'Académie, il était nécessaire de se poser la question des acteurs. Une exploitation des données recueillies dans les dépouillements a permis alors de mettre en évidence une population d'académiciens que j'ai appelée, faute de mieux, les académiciens technologues. Leur profil d'activité technicienne permet de les caractériser. Si l'exercice d'une commission d'examen est plus répandu dans la population académique que la production de mémoire technique, le croisement des deux m'a fait apparaître un groupe plus restreint, 23 académiciens plus tournés vers la technique et, en tête, Parent, Réaumur, Duhamel, Pitot et Hellot suivis de La Hire, Camus et Amontons, groupe qui, à l'évidence, montre un plus grand intérêt pour la technique que le reste du corps académique. Mais, le temps me manquait pour me lancer dans la prosopographie de ce groupe, puisque je voulais saisir la pensée commune du corps académique qui comme institution, suivant Mary Douglas, "pense » collectivement $^{8}$. C'est à travers les discours communs des uns et des autres, sans distinction 
systématique des auteurs de ces discours, que j'ai cherché à atteindre cette «doxa» sans cesse répétée d'une pensée de la technique conduite par la méthode scientifique et les mathématiques. Ces biographies sont d'ailleurs frustrantes, parfois seul l'éloge funèbre du secrétaire perpétuel nous donne à voir un académicien, ces éloges sont facilement accessibles et dans le cas des plus célèbres comme Réaumur, leur biographie a été faite. Je n'ai donné que quelques éléments de biographie pour Henri Pitot, souvenir personnel pour son loch et pour le canal du midi que j'ai connu étant enfant, il a une rue à son nom, entre autres, à Nîmes et à Carcassonne, rues modestes, quelque peu excentrées dans les deux cas.

Puisque j'avais entrepris de dépouiller systématiquement les registres de séances, je me devais de les croiser, dans la mesure du possible, « vers le haut » avec les histoires annuelles et « vers le bas » avec les pochettes de séances. C'est dans le registre de l'année 1747 et dans la pochette de séance de juillet de cette année, que j'ai découvert une " inconnue des archives », jamais encore, à ma connaissance, étudiée. La correspondance des acteurs principaux, l'accord entre l'Académie et la ville de Reims, étaient dans la pochette, il suffisait ensuite de tirer sur le fil. Sans grand espoir, craignant les destructions de la Grande Guerre, j'ai écrit aux archives municipales de Reims pour m'informer de l'existence d'archives relatives à cette école. Quinze jours plus tard, j'ai reçu une lettre de deux pages avec toutes les cotes concernant l'école, il suffisait, encore une fois, d'y passer deux jours pour compléter les sources de l'Académie, je les remercie encore de leur amabilité et de leur professionnalisme. Les acteurs de cette fondation se retrouvaient dans un réseau newtonien très représentatif, autour des Principia de Newton et de Madame du Chatelet. Au-delà du caractère singulier de cette fondation, les accords, les correspondances, le programme d'enseignement, les manuels montraient bien que ce que je qualifiais de pensée technique de l'Académie prenait corps dans un enseignement qui avait son soutien explicite. $\mathrm{Au}-$ delà de la fondation, dérogeant à mon cadre temporel, j'ai pu montrer la permanence de ces liens, la place prise par l'école de Reims comme école préparatoire aux écoles des armes savantes, Génie et Artillerie. Mais aussi l'attribution de brevet de maîtrise sans chef d'œuvre ouvrait une autre perspective sur la mise en place d'un enseignement technique d'un nouveau genre.

Il ne restait plus, c'est encore une litote, qu'à nouer ensemble tous les fils de ces chapitres pour tenter de caractériser cette pensée technique en la plaçant dans les régimes de la pensée opératoire. Encore technique selon les classifications d'AnneFrançoise Garçon mais déjà technologique, émergence d'une pensée que je pouvais corréler temporellement avec le début des écoles d'ingénieurs «à la française » où l'on expose les principes pour les réduire en pratique ou en exécution selon les mots du professeur de l'école de Reims. Une dure bataille avec le mot technologie dans toutes ses significations, a rendu nécessaire de faire des choix de sens, d'utilisation du mot au singulier ou au pluriel, de l'adjectif technologique plutôt que du mot pour, je l'espère, caractériser au mieux cette pensée technique de l'Académie royale des sciences.

$\mathrm{Si}$ je devais en quelques mots résumer les résultats de cette recherche, je mentionnerais d'abord la démonstration, quantitativement établie, de la place importante de la technique dans le travail académique. Au-delà de ce qui était connu, c'est-à-dire les examens d'inventions et les descriptions des arts, de grandes expertises techniques et un grand nombre d'étude techniques mettent en lumière ce travail interne de l'Académie sur la technique. Enfin, en réunissant les points communs de toutes ces modalités j'ai voulu montrer l'émergence d'un nouveau régime de pensée technique. Dans la continuité de la fondation de la science expérimentale du XVII ${ }^{e}$ siècle, les académiciens qui se réfèrent explicitement à Bacon, Galilée et Descartes, utilisent cette science pour 
conduire les techniques. Ce qu'il faut souligner dans cette rencontre entre la science moderne et les techniques, ce n'est pas tant la notion d'application de la science que l'utilisation de la méthode scientifique et des mathématiques. Ce que nous montrent les travaux techniques des académiciens, c'est la mise en œuvre d'une approche scientifique de la technique et la conséquence en est bien la nécessité de former des techniciens savants. On peut se demander alors si en regard nous ne voyons pas se former une autre figure, celle du savant technicien. Le conflit n'est pas noué dans la période qui nous intéresse mais la double élection de Jars et de Lavoisier en 1768 que le Roi ne veut pas trancher en les nommant tous les deux en est un signe.

$\mathrm{Au}$ terme de ce travail de recherche, si j'ai apporté quelques réponses à la question que je m'étais posée - pourquoi et comment l'Académie des sciences s'occupe-t-elle de technique ? - apparaissent de nouvelles questions, de nouvelles pistes de recherche. La plus naturelle d'abord : que ce passe-t-il après 1750 ? Des travaux importants existent, ceux d'Anne-Françoise Garçon, en particulier pour chimie et métallurgie autour d'Hellot et de Jars mais le dépouillement systématique reste à faire. Tout en étant quelque peu effrayé par les dizaines de milliers de pages restant à dépouiller, je vais essayer de poursuivre ce dépouillement, peut-être à un rythme différent. Autre piste : si comme je le pense, l'école de Reims a partiellement servi de classe préparatoire aux écoles de Mézières et de La Fère, alors le croisement des registres des élèves de Reims avec ceux de Mézières, déjà exploités mais à d'autres fins par Roger Chartier', doit permettre de l'établir encore plus fermement. Enfin, mais ici le questionnement et les problématiques sont encore un peu flous, j'ai vu apparaître de nombreux manuels destinés aux techniciens savants, aux ingénieurs militaires, Bélidor bien sûr, le plus connu, mais d'autres avec lui. Est-il possible d'en entreprendre une étude comparative de les replacer dans des échelles de niveau qui nous les rendraient plus concrets?

J'ai commencé cet exposé par une référence à ma vie professionnelle antérieure et je voudrais terminer par l'exergue d'un manuel technique contemporain qui marque bien cette tension permanente entre théorie et pratique dans le monde de la technique, vous pardonnerez certainement mon accent allemand :

"Nichts ist praktischer als eine gute Theorie ${ }^{10}$. »

Monsieur le président, mesdames et messieurs les membres du Jury, je vous remercie de votre attention.

${ }^{1}$ La soutenance a eu lieu à la Sorbonne, dans la salle Jean-
Baptiste Duroselle à laquelle on accède par la galerie citée.

2 GARÇON A-F., L’imaginaire et la pensée technique, Paris, Classiques Garnier, 2012.

3 VERIN H., La gloire des ingénieurs, L'intelligence technique du XVI e au XVIII ${ }^{e}$ siècle, Paris, Albin-Michel, 1993.

4 PICON A., L'invention de l'ingénieur moderne : l'Ecole des Ponts et Chaussées (1747-1851), Paris, Presses de l'Ecole nationale des Ponts et Chaussées, 1992.

5 RUSSO F., Introduction à l'histoire des techniques, Paris, Albert Blanchard, 1986.

${ }^{6}$ MAZAURIC S., Histoire des sciences à l'époque moderne, Paris, Armand Colin, 2009.

7 SCHLUTTER Christophe-André (traduction HELLOT Jean), De la fonte des mines, des fonderies et de ce qui en dépend, Tome premier, Paris, Pissot (veuve et fils), Hérissant, 1750. Tome second, 1753 .

8 DOUGLAS Mary, Comment pensent les institutions, Paris, La Découverte, 1999.

9 CHARTIER Roger, «Un recrutement scolaire au XVIII' siècle : l'Ecole royale du génie de Mézières », Revue d'histoire moderne et contemporaine, tome XX, juillet septembre 1973, pp. 353-375.

10 « Rien n'est plus pratique qu'une bonne théorie », dans GILLE J-C., DECAULNE P., et PELEGRIN M., Théorie et calcul des asservissements linéaires, Dunod, Paris, 1971. L'exergue est attribué à LEONHARD A. (spécialiste allemand des asservissements), sans autre référence. 